\title{
ESTRUTURA DO SUB-BOSQUE HERBÁCEO-ARBUSTIVO DA MATA DA SILVICULTURA, UMA FLORESTA ESTACIONAL SEMIDECIDUAL NO MUNICÍPIO DE VIÇOSA-MG ${ }^{1}$
}

\author{
João Augusto Alves Meira Neto² e Fernando Roberto Martins ${ }^{3}$
}

\begin{abstract}
RESUMO - Nos trabalhos em comunidades florestais, tradicionalmente são estudadas apenas a composição e a estrutura do componente arbóreo, relegando o estrato herbáceo-arbustivo ao esquecimento ou ao segundo plano. Os objetivos deste trabalho foram descrever a estrutura fitossociológica desse estrato para entender suas relações sinecológicas e, por fim, estudar a distribuição dos indivíduos pelas classes de tamanho para inferir sobre fatores e processos determinantes da organização florestal da Mata da Silvicultura. Para o estudo da fitossociologia da área foram utilizados os parâmetros de abundância obtidos a partir de $100 \mathrm{~m}^{2}$ de amostra subdividida em parcelas de $1 \mathrm{~m}^{2}$. A estrutura fitossociológica horizontal considerou todos os indivíduos com CAP menor que $10 \mathrm{~cm}$ ou com altura maior que $20 \mathrm{~cm}$. Os aspectos dinâmicos foram avaliados por meio da distribuição de tamanhos individuais expressos pelos diâmetros à altura do solo em cada população amostrada. Foram amostrados 1.193 indivíduos de 109 espécies, pertencentes a 41 famílias botânicas, resultando em um índice de diversidade de Shannon $\left(\mathrm{H}^{\prime}\right)$ de 3,38 e equabilidade $\left(\mathrm{J}^{\prime}\right)$ de 0,72 , valores considerados altos para a heterogeneidade do estrato herbáceo-arbustivo. As espécies mais importantes (VI) foram Piper lucaeanum, Psychotria conjugens, Olyra micrantha, Psychotria sessilis, Siparuna guianensis, Bambusa tuldoides, Ottonia leptostachya, Aparisthmium cordatum e Psychotria hastisepala. As famílias mais importantes (VI) foram Rubiaceae, Piperaceae, Poaceae, Monimiaceae, Leguminosae (Mimosoideae), Myrtaceae, Euphorbiaceae, Meliaceae, Lauraceae e Flacourtiaceae. Pela análise de distribuição de tamanhos foi levantada a hipótese de existirem dois grupos de espécies, segundo a estratégia que possuem de habitar o estrato herbáceo-arbustivo da Mata da Silvicultura. Um dos grupos seria formado pelas espécies que investem preferencialmente recursos energéticos no sistema caulinar e o outro, pelas espécies que investem recursos energéticos preferencialmente no sistema fotossintético.
\end{abstract}

Palavras-chave: Regeneração, fitossociologia de sub-bosque e estrutura de tamanhos.

\section{UNDERSTORY STRUCTURE OF SILVICULTURA FOREST, A SEASONAL TROPICAL FOREST IN VIÇOSA, BRAZIL}

\begin{abstract}
Forest community studies traditionally investigate arboreal structure and composition. Herb-shrub layers have little or no importance in these works. Initial information on herb-shrub layers of Brazilian forests was provided as a complement to arboreal studies. However, herb-shrub layers have recently gained a central importance in some works. The main objective of this study was to describe the phytosociological structure of the Silvicultura forest's herb-shrub layer to understand the synecological relations and study the populational dynamic processes involved. The phytosociological parameters used were obtained from $1 \mathrm{~m}^{2}$ split plots of $100 \mathrm{~m}^{2}$ samples. All individuals with less than $10 \mathrm{~cm} \mathrm{PBH} \mathrm{(perimeter} \mathrm{at} \mathrm{breast} \mathrm{height)} \mathrm{or} \mathrm{over} 20 \mathrm{~cm}$ were sampled. Dynamic processes were evaluated by using the size frequency distribution of sampled populations. The herb-shrub phytosociological structure consisted of 1193 individuals of 109 species of 41 families in samples of $100 \mathrm{~m}^{2}$. The Shannon diversity index $\left(H^{\prime}\right)$ was equal to 3.38 nats/individual and the equability $\left({ }^{\prime}\right)$ was equal to 0.72 , both
\end{abstract}

1 Recebido para publicação em 10.4.2001.

Aceito para publicação em 9.9.2003.

Parte da tese apresentada pelo primeiro autor ao Curso de Doutorado em Ciências Biológicas da UNICAMP.

2 Departamento de Biologia Vegetal da Universidade Federal de Viçosa - UFV, 36571-000 Viçosa-MG. Bolsista CAPES e CNPq, <j.meira@mail.ufv.br>. ${ }^{3}$ Departamento de Botânica da Universidade Estadual de Campinas - UNICAMP, Caixa Postal 6109, 13083-970 Campinas-SP, <fmartins@unicamp.br>. 
showing high heterogeneity under herb-shrub conditions. The most important species (VI) were Piper lucaeanum, Psychotria conjugens, Olyra micrantha, Psychotria sessilis, Siparuna guianensis, Bambusa tuldoides, Ottonia leptostachya, Aparisthmium cordatum and Psychotria hastisepala. The most important (VI) families were Rubiaceae, Piperaceae, Poaceae, Monimiaceae, Leguminosae (Mimosoideae), Myrtaceae, Euphorbiaceae, Meliaceae, Lauraceae and Flacourtiaceae. The size frequency distribution analysis raised the hypothesis that there were two species groups, with different strategies. One group would consist of species allocating photosynthates preferentially to their photosynthetic system, and the other group of species allocating photosynthates preferentially to their trunks.

Key words: Regeneration, understory structure, and size structure.

\section{INTRODUÇÃOO}

Os estudos florísticos e fitossociológicos em florestas de todo o mundo geralmente enfatizam o componente arbóreo, que é o principal detentor da biomassa florestal e se destaca pela importância econômica. Citadini-Zanete \& Baptista (1989) mostraram que no Brasil essa ênfase se repetiu nos estudos florísticos e fitossociológicos florestais. Os autores relataram que a relevância dos estudos das espécies florestais de menor porte está na pronunciada sensibilidade que manifestam pelas variações microclimáticas e edáficas. Contudo, a maioria dos estudos fitossociológicos de espécies arbustivas ou herbáceas florestais as circunscreve como item secundário, complementar, na caracterização dessas comunidades.

Vários trabalhos demonstraram a estrutura dos estratos inferiores de florestas brasileiras e paraguaias ocorrentes em amostras fitossociológicas (Cain et al., 1956; Takeuchi, 1960; Veloso \& Klein, 1963; Baptista \& Irgang, 1972; Lindeman et al., 1975; Knob, 1978; Schlittler, 1984; Cestaro et al., 1986; Citadini-Zanete \& Baptista, 1989; Stutz de Ortega, 1987, 1990; Andrade, 1992; Bernacci, 1992; Oliveira-Filho et al., 1994; Zickel, 1995; Meira-Neto \& Martins, 2000). Desses, os trabalhos de Schlittler (1984), Cestaro et al. (1986), Citadini-Zanete \& Baptista (1989); Andrade (1992), Bernacci (1992), Zickel (1995) e Meira-Neto \& Martins (2000) tiveram como objetivos principais o estudo do estrato herbáceoarbustivo em florestas. Portanto, ainda são poucos os trabalhos que têm como objetivos conhecer a composição florística e a estrutura do estrato herbáceo-arbustivo de uma floresta estacional semidecidual. Tal conhecimento é de fundamental importância para o entendimento da estrutura florestal como um todo, especialmente nos estudos de regeneração natural, de sucessão e de dinâmica de populações de plantas.

Os objetivos deste trabalho foram conhecer e estudar a estrutura fitossociológica e diamétrica do componente herbáceo-arbustivo da Mata da Silvicultura, como subsídio aos estudos de regeneração natural em Florestas Estacionais Semideciduais.

\section{MATERIAL E MÉTODOS}

\section{1. Área de Estudos}

O município de Viçosa situa-se nas coordenadas $20^{\circ} 45^{\prime} \mathrm{S}$ e $42^{\circ} 55^{\prime} \mathrm{W}$, perifericamente no norte da Zona da Mata, entrecortado por rios e córregos tributários do rio Doce (Valverde 1958, Mariscal-Flores 1993). O centro da cidade de Viçosa tem altitude de $650 \mathrm{~m}$, mas são encontradas altitudes maiores que $800 \mathrm{~m}$ nos topos dos muitos morros do município (Comissão Geográfica e Geológica de MInas Gerais, 1930).

O clima é tropical de altitude com verões chuvosos e invernos frios e secos, do tipo Cwb pelo sistema de Köppen (Valverde, 1958; Golfari, 1975; Departamento Nacional de Meteorologia, 1992). A precipitação média anual é de 1.221,4 mm (Departamento Nacional de Meteorologia, 1992).

A Mata da Silvicultura, local onde foi realizado este estudo, situa-se em altitudes entre 670 e $730 \mathrm{~m}$ e é um fragmento florestal de 17 ha resultante de regeneração natural. Foi adquirida pela Universidade Federal de Viçosa em 1936 e, desde então, encontra-se protegida de cortes e extração de madeira (Mariscal-Flores, 1993), o que tem garantido a manutenção do adiantado estádio sucessional, que conta atualmente com mais de 60 anos.

\subsection{Fitossociologia}

A amostra fitossociológica do componente herbáceo-arbustivo totalizou $100 \mathrm{~m}^{2}$. Cada uma das 100 unidades amostrais de $1 \mathrm{~m}^{2}(1 \mathrm{x} 1 \mathrm{~m})$ foi alocada no centro geométrico das parcelas da amostra fitossociológica do componente arbóreo (10 × $10 \mathrm{~m})$, organizada 
em blocos de $20 \times 50 \mathrm{~m}$. Os blocos foram alocados subjetivamente ao longo da área do fragmento, para não haver trechos extensos sem unidades amostrais. Dentro dos blocos a disposição foi sistemática.

Foram amostrados todos os indivíduos com circunferência à altura do peito (CAP) menor que $10 \mathrm{~cm}$ e com altura maior que $20 \mathrm{~cm}$.

Os parâmetros fitossociológicos calculados foram densidade, freqüência e dominância absolutas e relativas e, a partir dessas, o índice de valor de importância (VI), utilizando as seguintes fórmulas (Mueller-Dombois \& Ellenberg, 1974; Martins, 1991):

$$
\begin{aligned}
& \text { Densidade absoluta }(\mathrm{DA})=\mathrm{ni} / \mathrm{A} . \\
& \text { Densidade relativa }(\mathrm{DR})=100 \mathrm{ni} / \mathrm{N} . \\
& \text { Dominância absoluta }(\mathrm{DoA})=\mathrm{ABi} / \mathrm{A} . \\
& \text { Dominância relativa }(\mathrm{DoR})=100 \mathrm{ABi} / \mathrm{ABT} \text {. } \\
& \text { Frequiência absoluta }(\mathrm{FA})=100 \mathrm{Ui} / \mathrm{UT} . \\
& \text { Frequiência relativa }(\mathrm{FR})=100 \mathrm{FA} / \Sigma \mathrm{FAi} . \\
& \text { Valor de importância }(\mathrm{VI})=\mathrm{DR}+\mathrm{FR}+\mathrm{DoR} .
\end{aligned}
$$

em que

ni = número de indivíduos da espécie $\mathrm{i}$;

$\mathrm{N}=$ número de indivíduos amostrados;

$\mathrm{A}=$ área da amostra (em hectares);

$\mathrm{ABi}$ = área basal da espécie $\mathrm{i}$, obtida da soma das áreas basais individuais a partir da fórmula do círculo $\left(\mathrm{em} \mathrm{m}^{2}\right)$;

$\mathrm{ABT}=$ área basal total amostrada $\left(\mathrm{em} \mathrm{m}^{2}\right)$;

$\mathrm{Ui}=$ número de unidades amostrais com a ocorrência da espécie i; e

$\mathrm{UT}=$ número total de unidades amostrais.

Os parâmetros relativos e o VI foram calculados, colocando-se as famílias botânicas no lugar das espécies, com os mesmos objetivos.

Foram calculados o índice de diversidade de Shanon (H') e a equabilidade (J) para a amostra (Pielou, 1975).

Para calcular os referidos parâmetros, utilizou-se o software Fitopac 1 (Shepherd, 1994).

\subsection{Estrutura Diamétrica}

A distribuição diamétrica foi feita mediante o cômputo dos indivíduos amostrados de cada espécie dentro da classe diamétrica a que pertencessem (Harper, 1977). As classes de diâmetro foram estabelecidas com amplitude de $1 \mathrm{~cm}$, a partir do diâmetro mínimo de $0,1 \mathrm{~cm}$.

Para obtenção dos quadros de distribuição diamétrica foi utilizado o software Diamfito (Mota, 1995).

O quadro florístico das espécies amostradas está publicado em Meira Neto \& Martins (2000).

Para as plantas rizomatosas de maior densidade foram traçadas as curvas dos números de indivíduos pelo número de ramos aéreos que partem do rizoma. Esse procedimento leva em consideração o fato de que as ramificações do rizoma, segundo Harper (1977), podem servir como evidências morfológicas para determinação de idade em espécies herbáceas perenes.

\section{RESULTADOS E DISCUSSÃO}

\subsection{Fitossociologia}

Foram amostrados 1.193 indivíduos que perfizeram $0,07 \mathrm{~m}^{2}$ de área basal na amostra, o que confere uma densidade de 119.300 indivíduos e 7,07 m² de área basal por hectare.

O índice de diversidade de Shanon-Weaver $\left(\mathrm{H}^{\prime}\right)$ é 3,38 e a equabilidade (J) é 0,72 .

Tanto a densidade total, 119.300 indivíduos/ha, quanto o índice de diversidade de Shanon, 3,38, encontrados para a Mata da Silvicultura, assumiram valores próximos daqueles encontrados para os mesmos parâmetros na fazenda São Vicente, 110.900 indivíduos/ha e 3,50, respectivamente, em Campinas (Bernacci, 1992). Em Nova Lima (Andrade, 1992) foram encontrados densidade total de 304.000 indivíduos/ha e índice de diversidade de Shanon-Weaver de 3,87, valores superiores aos da Mata da Silvicultura em Viçosa. Essa diferença deve-se aos diferentes métodos de amostragem utilizados nos dois estudos em questão, uma vez que Andrade (1992) amostrou todos os indivíduos com menos de 1,2 m e não utilizou altura mínima para a amostragem.

As espécies que apresentam os maiores números de indivíduos e densidades absoluta e relativa são Piper lucaeanum, Olyra micrantha, Psychotria conjugens, Psychotria sessilis, Siparuna guianensis, Ottonia leptostachia, Psychotria hastisepala, Aparisthmium cordatum e Psychotria carthagenensis, decrescentemente (Quadro 1).

R. Árvore, Viçosa-MG, v.27, n.4, p.459-471, 2003 
Quadro 1 - Parâmetros fitossociológicos do estrato herbáceo-arbustivo da Mata da Silvicultura, Viçosa-MG. Densidade absoluta (DA), densidade relativa (DR), freqüência absoluta (FA), freqüência relativa (FR), dominância absoluta (DoA), dominância relativa (DoR) e valor de importância (VI)

Table 1 - Phytosociological parameters of the Silvicultura Forest understory, Viçosa, Brazil. Absolute density (DA), relative density (DR), absolute frequence (FA), relative frequency (FR), absolute dominancy (DoA), relative dominance (DoR), and importance value (VI)

\begin{tabular}{|c|c|c|c|c|c|c|c|}
\hline Espécie & $\begin{array}{c}\text { DA } \\
\text { (n/ha) }\end{array}$ & DR & FA & FR & $\begin{array}{c}\text { DoA } \\
\left(\mathrm{m}^{2} / \mathrm{ha}\right)\end{array}$ & DoR & VI \\
\hline Piper lucaeanum & $27.100,0$ & 22,72 & 18,00 & 3,07 & 0,4978 & 7,04 & 32,82 \\
\hline Psychotria conjugens & $8.000,0$ & 6,71 & 38,00 & 6,48 & 1,3029 & 18,42 & 31,61 \\
\hline Olyra micrantha & $17.800,0$ & 14,92 & 47,00 & 8,02 & 0,2961 & 4,19 & 27,13 \\
\hline Psychotria sessilis & $6.000,0$ & 5,03 & 32,00 & 5,46 & 0,7950 & 11,24 & 21,73 \\
\hline Siparuna guianensis & $3.800,0$ & 3,19 & 31,00 & 5,29 & 0,5447 & 7,70 & 16,17 \\
\hline Bambusa tuldoides & $1.000,0$ & 0,84 & 7,00 & 1,19 & 0,5853 & 8,27 & 10,31 \\
\hline Indeterminadas & $3.500,0$ & 2,93 & 24,00 & 4,10 & 0,1581 & 2,24 & 9,26 \\
\hline Ottonia leptostachya & $3.400,0$ & 2,85 & 20,00 & 3,41 & 0,0726 & 1,03 & 7,29 \\
\hline Aparisthimium cordatum & $2.100,0$ & 1,76 & 17,00 & 2,90 & 0,1772 & 2,50 & 7,17 \\
\hline Psychotria hastisepala & $2.200,0$ & 1,84 & 12,00 & 2,05 & 0,1290 & 1,82 & 5,72 \\
\hline Ixora bahiensis & $1.900,0$ & 1,59 & 16,00 & 2,73 & 0,0875 & 1,24 & 5,56 \\
\hline Psychotria carthagenensis & $1.900,0$ & 1,59 & 12,00 & 2,05 & 0,1217 & 1,72 & 5,36 \\
\hline Guarea pendula & 500,0 & 0,42 & 4,00 & 0,68 & 0,2732 & 3,86 & 4,96 \\
\hline Myrcia formosiana & $1.700,0$ & 1,42 & 14,00 & 2,39 & 0,0724 & 1,02 & 4,84 \\
\hline Rudgea lanceolata & $1.600,0$ & 1,34 & 12,00 & 2,05 & 0,0600 & 0,85 & 4,24 \\
\hline Casearia ulmifolia & 600,0 & 0,50 & 6,00 & 1,02 & 0,1840 & 2,60 & 4,13 \\
\hline Albizzia polycephala & $1.900,0$ & 1,59 & 12,00 & 2,05 & 0,0295 & 0,42 & 4,06 \\
\hline Sorocea bonplandii & $1.500,0$ & 1,26 & 10,00 & 1,71 & 0,0597 & 0,84 & 3,81 \\
\hline Pseudopiptadenia contorta & $1.100,0$ & 0,92 & 9,00 & 1,54 & 0,0546 & 0,77 & 3,23 \\
\hline Apuleia leiocarpa & 600,0 & 0,50 & 5,00 & 0,85 & 0,1312 & 1,85 & 3,21 \\
\hline Psychotria hygrophiloides & $1.800,0$ & 1,51 & 7,00 & 1,19 & 0,0200 & 0,28 & 2,99 \\
\hline Palicourea guianensis & 700,0 & 0,59 & 6,00 & 1,02 & 0,0643 & 0,91 & 2,52 \\
\hline Piper viçosanum & $1.100,0$ & 0,92 & 6,00 & 1,02 & 0,0320 & 0,45 & 2,40 \\
\hline Faramea multiflora & 700,0 & 0,59 & 6,00 & 1,02 & 0,0539 & 0,76 & 2,37 \\
\hline Calathea brasiliensis & 500,0 & 0,42 & 4,00 & 0,68 & 0,0842 & 1,19 & 2,29 \\
\hline Rhynchospora exaltata & 500,0 & 0,42 & 5,00 & 0,85 & 0,0678 & 0,96 & 2,23 \\
\hline Digitaria sanguinale & $1.200,0$ & 1,01 & 7,00 & 1,19 & 0,0019 & 0,03 & 2,23 \\
\hline Ocotea confusa & 800,0 & 0,67 & 7,00 & 1,19 & 0,0215 & 0,30 & 2,17 \\
\hline Heisteria silviani & $1.200,0$ & 1,01 & 5,00 & 0,85 & 0,0173 & 0,24 & 2,10 \\
\hline Lacistema pubescens & 900,0 & 0,75 & 7,00 & 1,19 & 0,0077 & 0,11 & 2,06 \\
\hline Prunus sellowii & 400,0 & 0,34 & 3,00 & 0,51 & 0,0856 & 1,21 & 2,06 \\
\hline Eugenia leptoclada & 700,0 & 0,59 & 6,00 & 1,02 & 0,0192 & 0,27 & 1,88 \\
\hline Copaifera langsdorffii & 600,0 & 0,50 & 5,00 & 0,85 & 0,0363 & 0,51 & 1,87 \\
\hline Chrysophyllum gonocarpum & 800,0 & 0,67 & 4,00 & 0,68 & 0,0235 & 0,33 & 1,69 \\
\hline Allophyllus sericeus & 700,0 & 0,59 & 4,00 & 0,68 & 0,0283 & 0,40 & 1,67 \\
\hline Anadenanthera colubrina & 700,0 & 0,59 & 5,00 & 0,85 & 0,0144 & 0,20 & 1,64 \\
\hline Mollinedia floribunda & 700,0 & 0,59 & 4,00 & 0,68 & 0,0214 & 0,30 & 1,57 \\
\hline Brosimum sellowii & 200,0 & 0,17 & 2,00 & 0,34 & 0,0726 & 1,03 & 1,54 \\
\hline Trichilia catigua & 500,0 & 0,42 & 5,00 & 0,85 & 0,0157 & 0,22 & 1,49 \\
\hline Ocotea odorifera & 500,0 & 0,42 & 4,00 & 0,68 & 0,0259 & 0,37 & 1,47 \\
\hline Wedelia paludosa & 700,0 & 0,59 & 4,00 & 0,68 & 0,0101 & 0,14 & 1,41 \\
\hline
\end{tabular}


Quadro 1, cont.

Table, cont.

\begin{tabular}{|c|c|c|c|c|c|c|c|}
\hline Espécie & $\begin{array}{c}\text { DA } \\
(\mathrm{n} / \mathrm{ha})\end{array}$ & DR & FA & FR & $\begin{array}{c}\text { DoA } \\
\left(\mathrm{m}^{2} / \mathrm{ha}\right)\end{array}$ & DoR & VI \\
\hline Tovomitopsis saldanhae & 300,0 & 0,25 & 2,00 & 0,34 & 0,0573 & 0,81 & 1,40 \\
\hline Beloperone monticola & 600,0 & 0,50 & 5,00 & 0,85 & 0,0024 & 0,03 & 1,39 \\
\hline Jacaranda macrantha & 500,0 & 0,42 & 5,00 & 0,85 & 0,0039 & 0,06 & 1,33 \\
\hline Ocotea pubescens & 400,0 & 0,34 & 4,00 & 0,68 & 0,0146 & 0,21 & 1,22 \\
\hline Trattinnickia ferruginea & 200,0 & 0,17 & 2,00 & 0,34 & 0,0503 & 0,71 & 1,22 \\
\hline Miconia sellowiana & 400,0 & 0,34 & 4,00 & 0,68 & 0,0132 & 0,19 & 1,20 \\
\hline Psychotria malaneoides & 600,0 & 0,50 & 2,00 & 0,34 & 0,0251 & 0,36 & 1,20 \\
\hline Inga cylindrica & 500,0 & 0,42 & 4,00 & 0,68 & 0,0049 & 0,07 & 1,17 \\
\hline Maytenus robusta & 400,0 & 0,34 & 4,00 & 0,68 & 0,0093 & 0,13 & 1,15 \\
\hline Inga affinis & 400,0 & 0,34 & 4,00 & 0,68 & 0,0076 & 0,11 & 1,13 \\
\hline Eugenia stictosepala & 300,0 & 0,25 & 2,00 & 0,34 & 0,0375 & 0,53 & 1,12 \\
\hline Machaerium brasiliense & 300,0 & 0,25 & 3,00 & 0,51 & 0,0240 & 0,34 & 1,10 \\
\hline Eugenia cf. gardneriana & 400,0 & 0,34 & 4,00 & 0,68 & 0,0052 & 0,07 & 1,09 \\
\hline Myrsine umbellata & 100,0 & 0,08 & 1,00 & 0,17 & 0,0573 & 0,81 & 1,06 \\
\hline Piptadenia gonoacantha & 400,0 & 0,34 & 3,00 & 0,51 & 0,0128 & 0,18 & 1,03 \\
\hline Thelypteris dentata & 200,0 & 0,17 & 2,00 & 0,34 & 0,0348 & 0,49 & 1,00 \\
\hline Miconia aff. pseudonervosa & 300,0 & 0,25 & 3,00 & 0,51 & 0,0122 & 0,17 & 0,94 \\
\hline Carpotroche brasiliensis & 300,0 & 0,25 & 3,00 & 0,51 & 0,0114 & 0,16 & 0,92 \\
\hline Costus spiralis & 200,0 & 0,17 & 2,00 & 0,34 & 0,0267 & 0,38 & 0,89 \\
\hline Platypodium elegans & 300,0 & 0,25 & 3,00 & 0,51 & 0,0079 & 0,11 & 0,88 \\
\hline Attalea dubia & 300,0 & 0,25 & 2,00 & 0,34 & 0,0196 & 0,28 & 0,87 \\
\hline Pteris denticulata & 500,0 & 0,42 & 2,00 & 0,34 & 0,0072 & 0,10 & 0,86 \\
\hline Machaerium floridum & 200,0 & 0,17 & 2,00 & 0,34 & 0,0240 & 0,34 & 0,85 \\
\hline Marlierea sp. & 200,0 & 0,17 & 2,00 & 0,34 & 0,0232 & 0,33 & 0,84 \\
\hline Swartzia elegans & 300,0 & 0,25 & 3,00 & 0,51 & 0,0045 & 0,06 & 0,83 \\
\hline Maytenus aquifolium & 300,0 & 0,25 & 3,00 & 0,51 & 0,0036 & 0,05 & 0,81 \\
\hline Mabea fistulifera & 300,0 & 0,25 & 3,00 & 0,51 & 0,0035 & 0,05 & 0,81 \\
\hline Allophyllus edulis & 300,0 & 0,25 & 3,00 & 0,51 & 0,0032 & 0,05 & 0,81 \\
\hline Lauraceae sp.1 & 300,0 & 0,25 & 2,00 & 0,34 & 0,0138 & 0,19 & 0,79 \\
\hline Casearia decandra & 100,0 & 0,08 & 1,00 & 0,17 & 0,0346 & 0,49 & 0,74 \\
\hline Geissomeria longiflora & 200,0 & 0,17 & 2,00 & 0,34 & 0,0155 & 0,22 & 0,73 \\
\hline Maranta ruiziana & 300,0 & 0,25 & 2,00 & 0,34 & 0,0078 & 0,11 & 0,70 \\
\hline Bathysa nicholsonii & 100,0 & 0,08 & 1,00 & 0,17 & 0,0314 & 0,44 & 0,70 \\
\hline Anemia phyllitidis & 300,0 & 0,25 & 2,00 & 0,34 & 0,0064 & 0,09 & 0,68 \\
\hline Pteris decurrens & 200,0 & 0,17 & 2,00 & 0,34 & 0,0118 & 0,17 & 0,68 \\
\hline Vernonia diffusa & 200,0 & 0,17 & 2,00 & 0,34 & 0,0083 & 0,12 & 0,63 \\
\hline Rubiaceae sp.1 & 200,0 & 0,17 & 2,00 & 0,34 & 0,0067 & 0,09 & 0,60 \\
\hline Miconia latecrenata & 200,0 & 0,17 & 2,00 & 0,34 & 0,0053 & 0,08 & 0,58 \\
\hline Nectandra psammophila & 200,0 & 0,17 & 2,00 & 0,34 & 0,0016 & 0,02 & 0,53 \\
\hline Machaerium stipitatum & 200,0 & 0,17 & 2,00 & 0,34 & 0,0010 & 0,01 & 0,52 \\
\hline Maranta bicolor & 200,0 & 0,17 & 2,00 & 0,34 & 0,0010 & 0,01 & 0,52 \\
\hline Rudgea leiocarpoides & 200,0 & 0,17 & 2,00 & 0,34 & 0,0006 & 0,01 & 0,52 \\
\hline Pteridophyta indet. . & 300,0 & 0,25 & 1,00 & 0,17 & 0,0016 & 0,02 & 0,45 \\
\hline
\end{tabular}

Continued.. 
Quadro 1, cont.

Table, cont.

\begin{tabular}{|c|c|c|c|c|c|c|c|}
\hline Espécie & $\begin{array}{c}\text { DA } \\
(\mathrm{n} / \mathrm{ha})\end{array}$ & DR & FA & FR & $\begin{array}{c}\text { DoA } \\
\left(\mathrm{m}^{2} / \mathrm{ha}\right)\end{array}$ & DoR & VI \\
\hline Bromelia balansae & 100,0 & 0,08 & 1,00 & 0,17 & 0,0079 & 0,11 & 0,37 \\
\hline Miconia tristis & 100,0 & 0,08 & 1,00 & 0,17 & 0,0064 & 0,09 & 0,34 \\
\hline Miconia mendoncaei & 100,0 & 0,08 & 1,00 & 0,17 & 0,0064 & 0,09 & 0,34 \\
\hline Aspidosperma polyneuron & 100,0 & 0,08 & 1,00 & 0,17 & 0,0064 & 0,09 & 0,34 \\
\hline Psychotria mapourioides & 100,0 & 0,08 & 1,00 & 0,17 & 0,0050 & 0,07 & 0,33 \\
\hline Mollinedia micrantha & 100,0 & 0,08 & 1,00 & 0,17 & 0,0050 & 0,07 & 0,33 \\
\hline Rollinia sylvatica & 100,0 & 0,08 & 1,00 & 0,17 & 0,0028 & 0,04 & 0,29 \\
\hline Solanum cernuиm & 100,0 & 0,08 & 1,00 & 0,17 & 0,0028 & 0,04 & 0,29 \\
\hline Erythroxylum pelleterianum & 100,0 & 0,08 & 1,00 & 0,17 & 0,0028 & 0,04 & 0,29 \\
\hline Ossaeamarginata & 100,0 & 0,08 & 1,00 & 0,17 & 0,0023 & 0,03 & 0,29 \\
\hline Licania spicata & 100,0 & 0,08 & 1,00 & 0,17 & 0,0020 & 0,03 & 0,28 \\
\hline Machaerium aculeatum & 100,0 & 0,08 & 1,00 & 0,17 & 0,0020 & 0,03 & 0,28 \\
\hline Psychotria myriantha & 100,0 & 0,08 & 1,00 & 0,17 & 0,0020 & 0,03 & 0,28 \\
\hline Rhynchospora sp. & 100,0 & 0,08 & 1,00 & 0,17 & 0,0017 & 0,02 & 0,28 \\
\hline Matayba elaeagnoides & 100,0 & 0,08 & 1,00 & 0,17 & 0,0013 & 0,02 & 0,27 \\
\hline Piper arboreum & 100,0 & 0,08 & 1,00 & 0,17 & 0,0013 & 0,02 & 0,27 \\
\hline Axonopus capilaris & 100,0 & 0,08 & 1,00 & 0,17 & 0,0010 & 0,01 & 0,27 \\
\hline Inga laurina & 100,0 & 0,08 & 1,00 & 0,17 & 0,0007 & 0,01 & 0,26 \\
\hline Miconia sp.2 & 100,0 & 0,08 & 1,00 & 0,17 & 0,0007 & 0,01 & 0,26 \\
\hline Dalbergia nigra & 100,0 & 0,08 & 1,00 & 0,17 & 0,0007 & 0,01 & 0,26 \\
\hline Casearia sylvestris & 100,0 & 0,08 & 1,00 & 0,17 & 0,0007 & 0,01 & 0,26 \\
\hline Xylopia sericea & 100,0 & 0,08 & 1,00 & 0,17 & 0,0007 & 0,01 & 0,26 \\
\hline Qualea jundiahy & 100,0 & 0,08 & 1,00 & 0,17 & 0,0003 & 0,01 & 0,26 \\
\hline Psychotria tenerior & 100,0 & 0,08 & 1,00 & 0,17 & 0,0003 & 0,01 & 0,26 \\
\hline Campomanesia xanthocarpa & 100,0 & 0,08 & 1,00 & 0,17 & 0,0003 & 0,01 & 0,26 \\
\hline
\end{tabular}

As espécies que ocorrem no maior número de unidades amostrais e, conseqüentemente, têm as maiores frequiências absoluta e relativa, em ordem decrescente, são Olyra micrantha, Psychotria conjugens, Psychotria sessilis, Siparuna guianensis, Ottonia leptostachya, Piper lucaeanum, Aparisthmium cordatum, Rubiaceae sp.1 e Myrcia formosiana (Quadro 1).

Em ordem decrescente, as espécies que apresentaram maiores áreas basais e dominâncias absolutas e relativas são Psychotria conjugens, Psychotria sessilis, Bambusa tuldoides, Siparuna guianensis, Piper lucaeanum, Olyra micrantha, Guarea pendula e Aparisthmium cordatum (Quadro 1).

As principais espécies em índice de valor de importância (VI) são, em ordem decrescente, Piper lucaeanum, Psychotria conjugens, Olyra micrantha, Psychotria sessilis, Siparuna guianensis, Bambusa tuldoides, Ottonia leptostachya, Aparisthmiuim cordatum e Psychotria hastisepala (Quadro 1).

As principais famílias em índice de valor de importância (VI) são Rubiaceae, Piperaceae, Poaceae, Monimiaceae, Leguminosae (Mimosoideae), Myrtaceae, Euphorbiaceae, Meliaceae, Lauraceae e Flacourtiaceae (Quadro 2).

A amostra evidenciou que a família Rubiaceae, a mais rica em espécies, também tem destaque fitossociológico, com espécies entre as mais densas, freqüentes e dominantes, sendo a família com maior índice de valor de importância (VI). As espécies mais representativas da família são do gênero Psychotria. Psychotria conjugens e Psychotria sessilis são as espécies que mostraram maior dominância. 
Quadro 2 - Parâmetros fitossociológicos das famílias do estrato herbáceo-arbustivo da Mata da Silvicultura, Viçosa-MG. Número de indivíduos $(\mathrm{N})$, densidade relativa $(\mathrm{DR})$, freqüência relativa $(\mathrm{FR})$, dominância relativa $(\mathrm{DoR})$ e valor de importância (VI)

Table 2 - Phytosociological parameters of plant families of the Silvicultura Forest understory, Viçosa, Brazil. Number of plants $(N)$, relative density $(D R)$, relative frequency $(F R)$, relative dominance $(D o R)$ and importance value (VI)

\begin{tabular}{|c|c|c|c|c|c|}
\hline Família & $\mathrm{N}$ & $\mathrm{DR}(\%)$ & FR $(\%)$ & $\operatorname{DoR}(\%)$ & VI \\
\hline Rubiaceae & 262 & 21,96 & 16,38 & 38,24 & 76,59 \\
\hline Piperaceae & 317 & 26,57 & 7,23 & 8,53 & 42,34 \\
\hline Poaceae & 201 & 16,85 & 11,49 & 12,50 & 40,84 \\
\hline Monimiaceae & 46 & 3,86 & 7,02 & 8,07 & 18,95 \\
\hline Leguminosae (Mimosoideae) & 51 & 4,27 & 5,96 & 1,76 & 11,99 \\
\hline Indeterminadas & 38 & 3,19 & 5,32 & 2,26 & 10,76 \\
\hline Myrtaceae & 34 & 2,85 & 5,53 & 2,23 & 10,61 \\
\hline Euphorbiaceae & 24 & 2,01 & 4,04 & 2,55 & 8,61 \\
\hline Meliaceae & 10 & 0,84 & 1,91 & 4,08 & 6,84 \\
\hline Lauraceae & 22 & 1,84 & 3,62 & 1,09 & 6,55 \\
\hline Flacourtiaceae & 11 & 0,92 & 2,13 & 3,26 & 6,31 \\
\hline Moraceae & 17 & 1,42 & 2,55 & 1,87 & 5,85 \\
\hline Caesalpiniaceae & 12 & 1,01 & 2,13 & 2,37 & 5,50 \\
\hline Leguminosae (Faboideae) & 15 & 1,26 & 2,98 & 0,90 & 5,14 \\
\hline Melastomataceae & 13 & 1,09 & 2,55 & 0,66 & 4,30 \\
\hline Marantaceae & 10 & 0,84 & 1,70 & 1,31 & 3,85 \\
\hline Sapindaceae & 11 & 0,92 & 1,70 & 0,46 & 3,09 \\
\hline Cyperaceae & 6 & 0,50 & 1,28 & 0,98 & 2,76 \\
\hline Lacistemataceae & 9 & 0,75 & 1,49 & 0,11 & 2,35 \\
\hline Olacaceae & 12 & 1,01 & 1,06 & 0,24 & 2,31 \\
\hline Compositae & 9 & 0,75 & 1,28 & 0,26 & 2,29 \\
\hline Celastraceae & 7 & 0,59 & 1,49 & 0,18 & 2,26 \\
\hline Acanthaceae & 8 & 0,67 & 1,28 & 0,25 & 2,20 \\
\hline Rosaceae & 4 & 0,34 & 0,64 & 1,21 & 2,18 \\
\hline Sapotaceae & 8 & 0,67 & 0,85 & 0,33 & 1,85 \\
\hline Pteridaceae & 7 & 0,59 & 0,85 & 0,27 & 1,71 \\
\hline Bignoniaceae & 5 & 0,42 & 1,06 & 0,06 & 1,54 \\
\hline Guttiferae & 3 & 0,25 & 0,43 & 0,81 & 1,49 \\
\hline Burseraceae & 2 & 0,17 & 0,43 & 0,71 & 1,30 \\
\hline Myrsinaceae & 1 & 0,08 & 0,21 & 0,81 & 1,11 \\
\hline Thelypteridaceae & 2 & 0,17 & 0,43 & 0,49 & 1,09 \\
\hline Costaceae & 2 & 0,17 & 0,43 & 0,38 & 0,97 \\
\hline Arecaceae & 3 & 0,25 & 0,43 & 0,28 & 0,95 \\
\hline Schyzaeaceae & 3 & 0,25 & 0,43 & 0,09 & 0,77 \\
\hline Annonaceae & 2 & 0,17 & 0,43 & 0,05 & 0,64 \\
\hline Bromeliaceae & 1 & 0,08 & 0,21 & 0,11 & 0,41 \\
\hline Apocynaceae & 1 & 0,08 & 0,21 & 0,09 & 0,39 \\
\hline Erythroxylaceae & 1 & 0,08 & 0,21 & 0,04 & 0,34 \\
\hline Solanaceae & 1 & 0,08 & 0,21 & 0,04 & 0,34 \\
\hline Chrysobalanaceae & 1 & 0,08 & 0,21 & 0,03 & 0,32 \\
\hline Vochysiaceae & 1 & 0,08 & 0,21 & 0,00 & 0,30 \\
\hline
\end{tabular}




\subsection{Distribuição Diamétrica}

A distribuição dos indivíduos de cada espécie pelas classes diamétricas está representada no Quadro 3.

Com poucas exceções, as curvas de distribuição diamétrica são unimodais, com as classes de menores diâmentros possuindo maior número de indivíduos. Entretanto, podem ser observados dois padrões entre as espécies com maior densidade. Um dos padrões apresenta a grande maioria dos indivíduos na primeira classe diamétrica (de 0,1 a $1,0 \mathrm{~cm}$ ), devendo ser ressaltado que poucos ou nenhum indivíduo aparece nas classes seguintes. Exemplos desse padrão são Piper lucaeanum, Olyra micrantha, Ottonia leptostachya, Albizzia polycephala, Psychotria higrophyloides, Digitaria sanguinalis e Heisteria silviani. O outro padrão encontrado apresenta maior número de indivíduos na classe de menores diâmetros, e nas classes seguintes encontra-se menor número de indivíduos. $\mathrm{O}$ formato da curva para esse padrão é de "J"-invertido. As espécies que se enquadram nesse padrão são Psychotria conjugens, Psychotria sessilis, Siparuna guianensis, Aparisthmium cordatum, Psychotria carthagenesis, Myrcia formosiana, Rudgea lanceolata e Sorocea bonplandii (Quadro 3).

Quadro 3 - Distribuição diamétrica das espécies do estrato herbáceo-arbustivo da Mata da Silvicultura, Viçosa-MG Table 3 -Diametric distribution of understory species of the Silvicultura forest, Viçosa-MG

\begin{tabular}{|c|c|c|c|c|c|c|c|c|c|}
\hline \multirow{2}{*}{ Espécie } & \multicolumn{8}{|c|}{ Centro de Classe $(\mathrm{cm})$} & \multirow{2}{*}{ Total } \\
\hline & 0,5 & 1,5 & 2,5 & 3,5 & 4,5 & 5,5 & 6,5 & 7,5 & \\
\hline Piper lucaeanum & 269 & 2 & & & & & & & 271 \\
\hline Olyra micrantha & 175 & 2 & & 1 & & & & & 178 \\
\hline Psychotria conjugens & 42 & 28 & 7 & 2 & & 1 & & & 80 \\
\hline Psychotria sessilis & 38 & 16 & 3 & 2 & 1 & & & & 60 \\
\hline Siparuna guianensis & 26 & 8 & 2 & 1 & 1 & & & & 38 \\
\hline Indeterminadas & 29 & 5 & 1 & & & & & & 35 \\
\hline Ottonia leptostachya & 34 & & & & & & & & 34 \\
\hline Psychotria hastisepala & 13 & 9 & & & & & & & 22 \\
\hline Aparisthimium cordatum & 16 & 3 & 2 & & & & & & 21 \\
\hline Ixora bahiensis & 17 & 1 & 1 & & & & & & 19 \\
\hline Psychotria carthagenensis & 14 & 5 & & & & & & & 19 \\
\hline Albizzia polycephala & 19 & & & & & & & & 19 \\
\hline Psychotria hygrophiloides & 18 & & & & & & & & 18 \\
\hline Myrcia formosiana & 14 & 3 & & & & & & & 17 \\
\hline Rudgea lanceolata & 13 & 3 & & & & & & & 16 \\
\hline Sorocea bonplandii & 13 & 2 & & & & & & & 15 \\
\hline Digitaria sanguinale & 12 & & & & & & & & 12 \\
\hline Heisteria silviani & 12 & & & & & & & & 12 \\
\hline Pseudopiptadenia contorta & 8 & 3 & & & & & & & 11 \\
\hline Piper viçosanum & 9 & 2 & & & & & & & 11 \\
\hline Bambusa tuldoides & 4 & 4 & 1 & & & & & 1 & 10 \\
\hline Lacistema pubescens & 9 & & & & & & & & 9 \\
\hline Ocotea confusa & 8 & & & & & & & & 8 \\
\hline Chrysophyllum gonocarpum & 7 & 1 & & & & & & & 8 \\
\hline Palicourea guianensis & 4 & 2 & 1 & & & & & & 7 \\
\hline Faramea multiflora & 6 & & 1 & & & & & & 7 \\
\hline Eugenia leptoclada & 6 & 1 & & & & & & & 7 \\
\hline Allophyllus sericeus & 6 & 1 & & & & & & & 7 \\
\hline Anadenanthera colubrina & 6 & 1 & & & & & & & 7 \\
\hline
\end{tabular}


Quadro 3, cont.

Table 3, cont.

\begin{tabular}{|c|c|c|c|c|c|c|c|c|c|}
\hline \multirow{2}{*}{ Espécie } & \multicolumn{8}{|c|}{ Centro de Classe $(\mathrm{cm})$} & \multirow{2}{*}{ Total } \\
\hline & 0,5 & 1,5 & 2,5 & 3,5 & 4,5 & 5,5 & 6,5 & 7,5 & \\
\hline Mollinedia floribunda & 6 & 1 & & & & & & & 7 \\
\hline Wedelia paludosa & 7 & & & & & & & & 7 \\
\hline Casearia ulmifolia & 4 & 1 & & & 1 & & & & 6 \\
\hline Apuleia leiocarpa & 2 & 3 & & 1 & & & & & 6 \\
\hline Copaifera langsdorffii & 4 & 2 & & & & & & & 6 \\
\hline Beloperone monticola & 6 & & & & & & & & 6 \\
\hline Psychotria malaneoides & 5 & 1 & & & & & & & 6 \\
\hline Guarea pendula & & 2 & 2 & & 1 & & & & 5 \\
\hline Calathea brasiliensis & 3 & 1 & 1 & & & & & & 5 \\
\hline Rhynchospora exaltata & 3 & 1 & 1 & & & & & & 5 \\
\hline Trichilia catigua & 4 & 1 & & & & & & & 5 \\
\hline Ocotea odorifera & 3 & 2 & & & & & & & 5 \\
\hline Jacaranda macrantha & 5 & & & & & & & & 5 \\
\hline Inga cylindrica & 5 & & & & & & & & 5 \\
\hline Pteris denticulata & 5 & & & & & & & & 5 \\
\hline Prunus sellowii & 2 & 1 & & 1 & & & & & 4 \\
\hline Ocotea pubescens & 3 & 1 & & & & & & & 4 \\
\hline Miconia sellowiana & 3 & 1 & & & & & & & 4 \\
\hline Maytenus robusta & 4 & & & & & & & & 4 \\
\hline Inga affinis & 4 & & & & & & & & 4 \\
\hline Eugenia cf. gardneriana & 4 & & & & & & & & 4 \\
\hline Piptadenia gonoacantha & 4 & & & & & & & & 4 \\
\hline Tovomitopsis saldanhae & 1 & 1 & 1 & & & & & & 3 \\
\hline Eugenia stictosepala & 1 & 2 & & & & & & & 3 \\
\hline Machaerium brasiliense & 1 & 2 & & & & & & & 3 \\
\hline Miconia aff. pseudonervosa & 2 & 1 & & & & & & & 3 \\
\hline Carpotroche brasiliensis & 2 & 1 & & & & & & & 3 \\
\hline Attalea dubia & 2 & 1 & & & & & & & 3 \\
\hline Platypodium elegans & 3 & & & & & & & & 3 \\
\hline Swartzia elegans & 3 & & & & & & & & 3 \\
\hline Maytenus aquifolium & 3 & & & & & & & & 3 \\
\hline Mabea fistulifera & 3 & & & & & & & & 3 \\
\hline Allophyllus edulis & 3 & & & & & & & & 3 \\
\hline Lauraceae sp.1 & 2 & 1 & & & & & & & 3 \\
\hline Maranta ruiziana & 3 & & & & & & & & 3 \\
\hline Anemia phyllitidis & 3 & & & & & & & & 3 \\
\hline Pteridophyta indet & 3 & & & & & & & & 3 \\
\hline Brosimum sellowii & 1 & & & 1 & & & & & 2 \\
\hline Trattinnickia ferruginea & 1 & & 1 & & & & & & 2 \\
\hline Thelypteris dentata & 1 & & 1 & & & & & & 2 \\
\hline Costus spiralis & 1 & 1 & & & & & & & 2 \\
\hline
\end{tabular}

Continua...

Continued... 
Quadro 3, cont.

Table 3, cont.

\begin{tabular}{|c|c|c|c|c|c|c|c|c|c|}
\hline \multirow{2}{*}{ Espécie } & \multicolumn{8}{|c|}{ Centro de Classe $(\mathrm{cm})$} & \multirow{2}{*}{ Total } \\
\hline & 0,5 & 1,5 & 2,5 & 3,5 & 4,5 & 5,5 & 6,5 & 7,5 & \\
\hline Machaerium floridum & 1 & 1 & & & & & & & 2 \\
\hline Marlierea sp. & & 2 & & & & & & & 2 \\
\hline Geissomeria longiflora & 1 & 1 & & & & & & & 2 \\
\hline Pteris decurrens & 1 & 1 & & & & & & & 2 \\
\hline Vernonia diffusa & 2 & & & & & & & & 2 \\
\hline Rubiaceae sp.1 & 2 & & & & & & & & 2 \\
\hline Miconia latecrenata & 2 & & & & & & & & 2 \\
\hline Nectandra psammophila & 2 & & & & & & & & 2 \\
\hline Machaerium stipitatum & 2 & & & & & & & & 2 \\
\hline Maranta bicolor & 2 & & & & & & & & 2 \\
\hline Rudgea leiocarpoides & 2 & & & & & & & & 2 \\
\hline Myrsine umbellata & & & 1 & & & & & & 1 \\
\hline Casearia decandra & & & 1 & & & & & & 1 \\
\hline Bathysa nicholsonii & & & 1 & & & & & & 1 \\
\hline Bromelia balansae & & 1 & & & & & & & 1 \\
\hline Aspidosperma polyneuron & 1 & & & & & & & & 1 \\
\hline Miconia mendoncaei & 1 & & & & & & & & 1 \\
\hline Miconia tristis & 1 & & & & & & & & 1 \\
\hline Psychotria mapourioides & 1 & & & & & & & & 1 \\
\hline Mollinedia micrantha & 1 & & & & & & & & 1 \\
\hline Solanum cernuum & 1 & & & & & & & & 1 \\
\hline Rollinia sylvatica & 1 & & & & & & & & 1 \\
\hline Erythroxylum pelleterianum & 1 & & & & & & & & 1 \\
\hline Ossaeamarginata & 1 & & & & & & & & 1 \\
\hline Licania spicata & 1 & & & & & & & & 1 \\
\hline Psychotria myriantha & 1 & & & & & & & & 1 \\
\hline Machaerium aculeatum & 1 & & & & & & & & 1 \\
\hline Rhynchospora sp. & 1 & & & & & & & & 1 \\
\hline Matayba elaeagnoides & 1 & & & & & & & & 1 \\
\hline Piper arboreum & 1 & & & & & & & & 1 \\
\hline Axonopus capilaris & 1 & & & & & & & & 1 \\
\hline Casearia sylvestris & 1 & & & & & & & & 1 \\
\hline Miconia sp.2 & 1 & & & & & & & & 1 \\
\hline Inga laurina & 1 & & & & & & & & 1 \\
\hline Dalbergia nigra & 1 & & & & & & & & 1 \\
\hline Xylopia sericea & 1 & & & & & & & & 1 \\
\hline Campomanesia xanthocarpa & 1 & & & & & & & & 1 \\
\hline Qualea jundiahy & 1 & & & & & & & & 1 \\
\hline Psychotria tenerior & 1 & & & & & & & & 1 \\
\hline Total & 1.013 & 136 & 29 & 9 & 4 & 1 & 0 & 1 & 1.193 \\
\hline
\end{tabular}


Quadro 4 - Distribuição do número de indivíduos das espécies rizomatosas pelo número de ramificações aéreas que partem do rizoma, na Mata da Silvicultura, Viçosa-MG

Table 4 - Distribution of number of plants of hemicryptophytic species by number of aerial branches from the subterraneous stems. Silvicultura Forest, Viçosa-MG

\begin{tabular}{|c|c|c|c|c|c|c|c|c|c|c|c|}
\hline \multirow{2}{*}{ Espécie } & \multicolumn{10}{|c|}{ Número de Ramos } & \multirow{2}{*}{ Total } \\
\hline & 1 & 2 & 3 & 4 & 5 & 6 & 7 & 8 & 9 & 10 & \\
\hline Piper lucaeanum & 124 & 96 & 33 & 11 & 4 & 1 & 1 & 0 & 0 & 1 & 271 \\
\hline Olyra micrantha & 64 & 45 & 33 & 18 & 10 & 6 & 1 & 1 & 0 & 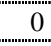 & 178 \\
\hline Ottonia leptostachya & 9 & 12 & 6 & 4 & 0 & 3 & 0 & 0 & 0 & 0 & 34 \\
\hline Digitaria sanguinale & 7 & 2 & 2 & 1 & 0 & 0 & 0 & 0 & 0 & 0 & 12 \\
\hline Bambusa tuldoides & 1 & 3 & 2 & 2 & 0 & 1 & 0 & 0 & 1 & 0 & 10 \\
\hline Total & 205 & 158 & 76 & 36 & 14 & 11 & 2 & 1 & 1 & 1 & 505 \\
\hline
\end{tabular}

Para as espécies rizomatosas que apresentaram dez indivíduos ou mais, foi elaborada uma distribuição do número de indivíduos pelo número de ramificações aéreas que partem do rizoma (Quadro 4). Piper lucaeanum, Digitaria sanguinalis e Olyra micrantha apresentaram curvas de distribuição com forma de "J"-invertido. Ottonia leptostachya e Bambusa tuldoides apresentaram distribuição descontínua, dando origem a curvas que não se enquadraram no padrão de "J"-invertido.

O conjunto das espécies que apresentaram distribuição diamétrica no padrão em que a grande maioria dos indivíduos se encontra na classe de menor diâmetro divide-se fundamentalmente em espécies rizomatosas e em indivíduos jovens e plântulas de espécies com caules aéreos. As rizomatosas têm, em geral, várias ramificações de pequeno diâmetro, que mesmo ao somar suas áreas basais o diâmetro correspondente de seus indivíduos continuará pertencendo à classe de menores diâmetros. Nesses casos, a estrutura diamétrica não nos dá nenhum indício da estrutura de tamanho de suas populações. Já as espécies com caules aéreos desse conjunto se constituem em interessante objeto de estudos populacionais nos quais seriam estudadas as causas desse tipo de distribuição. Uma das possíveis explicações para esse tipo de distribuição diamétrica entre as espécies com caules aéreos é a existência de bancos de plântulas que tenham crescimento muito lento em condições normais de subbosque e que respondam rapidamente às alterações ambientais que venham a alterar essa normalidade, beneficiando seus desenvolvimentos.

O conjuto de espécies que tiveram suas distribuições diamétricas originando curvas com forma de "J"-invertido é formado por espécies de sub-bosque do componente arbóreo. Como estão adaptadas às condições encontradas abaixo do dossel, a passagem de indivíduos de uma classe diamétrica para outra de diâmetros maiores é contínua, pois o crescimento dos indivíduos é constante nas condições de sub-bosque. O formato de "J"-invertido deve-se ao constante recrutamento e à taxa de mortalidade entre as classes.

As espécies rizomatosas não puderam ter suas estruturas de tamanho definidas pela análise de distribuição diamétrica. Mesmo com as limitações que apresentam, as análises de distribuições diamétricas fornecem importantes indícios a respeito das estruturas populacionais. Assim, a distribuição do número de ramificações aéreas que partem do rizoma foi muito elucidativo, mostrando que as mais densas podem ter suas estruturas de tamanho determinadas, como no caso de Piper lucaeanum, Olyra micrantha, Digitaria sanguinalis, Ottonia leptostachya e Bambusa tuldoides.

\section{CONCLUSÕES}

Piper lucaeanum, a espécie com maior densidade, não figura entre as mais freqüentes, o que evidencia uma distribuição agregada.

Olyra micrantha, a espécie com a segunda maior densidade, foi a que apresentou as mais altas frequiências, sendo uma espécie bem-distribuída pela área de amostragem.

Os taxa Rubiaceae e Psychotria são os maiores contribuintes em biomassa no componente herbáceoarbustivo da Mata da Silvicultura, respectivamente, em nível de família e de gênero.

A distribuição do número de ramificações aéreas que partem do rizoma mostrou que as espécies rizomatosas mais densas podem ter suas estruturas de tamanho determinadas, como no caso de Piper lucaeanum,

R. Árvore, Viçosa-MG, v.27, n.4, p.459-471, 2003 
Olyra micrantha, Digitaria sanguinalis, Ottonia leptostachya e Bambusa tuldoides.

Analisando as distribuições diamétrica e de ramificação de Olyra micrantha, nota-se que, apesar de a estrutura de tamanho ser definida pelo número de ramificações, a fase reprodutiva dessa espécie parece que só é alcançada quando seus indivíduos lançam ramos aéreos mais grossos. Observações de campo indicam que ramos mais grossos só são lançados em clareiras. Este parece ser interessante objeto de estudo populacional.

\section{REFERÊNCIAS BIBLIOGRÁFICAS}

ANDRADE, P. M. Estrutura do estrato herbáceo de trechos da Reserva Biológica Mata do Jambreiro, Nova Lima, MG. 1992. 90 f. Dissertação (Mestrado em Biologia Vegetal) - Universidade Estadual de Campinas, Campinas, 1992.

BAPTISTA, L. R. M.; IRGANG, B. Nota sobre a composição florística de uma comunidade florestal dos arredores de Porto Alegre. Iheringia, série Botânica, v. 16, p. 3-8, 1972.

BERNACCI, L. C. Estudo florístico e fitossociológico de uma floresta no município de Campinas, com ênfase nos componentes herbáceo e arbustivo. 1992. $146 \mathrm{f}$.

Dissertação (Mestrado em Biologia Vegetal) - Universidade Estadual de Campinas, Campinas, 1992.

CAIN, S. A. et al. Application of some phytosociological techniques to Brazilian rain forest. American Journal of Botany, v. 43, p. 911-41, 1956.

CESTARO, L. A.; WAECHTER, J. L.; BAPTISTA, L. R. M. Fitossociologia do estrato herbáceo da mata de araucária da Estação Ecológica de Aracuri, Esmeralda, RS. Hoehnea, v. 13, p. $59-72,1986$.

CITADINI-ZANETE, V.; BAPTISTA, L. R. M. Vegetação herbácea terrícola de uma comunidade florestal em Limoeiro, município de Torres, Rio Grande do Sul, Brasil. Boletim do Instituto de Biociências/UFRGS, v. 45, p. 187, 1989.

COMISSÃO GEOGRÁFICA E GEOLÓGICA DE MINAS GERAIS - CGGMG. Viçosa. Folha no 25 N1E3, Belo Horizonte: 1930. 1 mapa.

DEPARTAMENTO NACIONAL DE METEOROLOGIA DNM. Normais climatológicas (1961 - 1990). Brasília: 1992. $84 \mathrm{p}$.

R. Árvore, Viçosa-MG, v.27, n.4, p.459-471, 2003
GOLFARI, L. Zoneamento ecológico do Estado de Minas Gerais. Belo Horizonte: Centro de Pesquisa Florestal da Região do Cerrado, 1975. 65 p.

HARPER, J. L. Population Biology of plants. London: Academic Press, 1977. 891 p.

KNOB, A. Levantamento fitossociológico da formaçãomata do Morro do Coco, Viamão, RS, Brasil. Iheringia, série Botânica, v. 23, p. 65-108, 1978.

LINDEMAN, J. C. et al. Estudos botânicos no Parque Estadual de Torres, Rio Grande do Sul - Brasil; II -

Levantamento florístico da Planície do Curtume, da área de Itapeva e da área colonizada. Iheringia, série Botânica, v. 21, p. 15-52, 1975.

MARISCAL-FLORES, E. J. Potencial produtivo e alternativas de manejo sustentável de um fragmento de Mata Atlântica secundária, Município de Viçosa, Minas Gerais. 1993. 165 f. Dissertação (Mestrado em Ciências Florestais) - Universidade Federal de Viçosa, Viçosa, 1993.

MARTINS, F. R. Estrutura de uma floresta mesófila. Campinas: UNICAMP, 1991. 246 p.

MEIRA-NETO, J. A. A.; MARTINS, F. R. Composição florística do estrato herbáceo-arbustivo de uma floresta estacional semidecidual em Viçosa - MG. Revista Árvore, v. 24, n. 4 , p. $407-416,2000$.

MOTA, L. P. Diamfito. Programa de análise de distribuição diamétrica. Viçosa: Universidade Federal de Viçosa, 1995.

MUELLER-DOMBOIS, D.; ELLENBERG, H. Aims and methods of vegetation ecology. New York: John Wiley \& Sons, 1974. 547 p.

OLIVEIRA-FILHO, A. T. et al. Comparison of the woody flora and soils of six areas of montane semideciduous forest in southern Minas Gerais, Brazil. Edinburgh Journal of Botany, v. 51, n. 3, p. 355-89, 1994.

PIELOU, E. C. Ecological diversity. New York: John Wiley \& Sons, 1975. 165 p.

SCHLITTLER, F. H. M. Composição florística e estrutura fitossociológica do sub-bosque de uma plantação de Eucalyptus tereticornis Sm., no município de Rio Claro SP. 1984. 141 f. Dissertação (Mestrado em Ecologia) Universidade Estadual Paulista Júlio de Mesquita Filho, Rio Claro, 1984.

SHEPHERD, G. J. Fitopac 1. Manual do usuário. Campinas: UNICAMP, 1994. 90 p. 
STUTZ DE ORTEGA, L. C. Etudes floristiques de divers stades secondaires des formations forestières du Haut Parana (Paraguay Oriental). Structure, composition floristique et régénération naturelle: comparaison entre la forêt primaire et la forêt selectivement exploitée. Candollea, v. 42, p. 205-62, 1987.

STUTZ DE ORTEGA, L. C. Etudes floristiques de divers stades secondaires des formations forestières du Haut parana (Paraguay oriental). Structure, composition floristique et recrû forêtier: analyse de cinq stades de succession secondaire. Candollea, v. 45, p. 81-123, 1990.

TAKEUCHI, M. Estrutura da vegetação na Amazônia III. A mata de campina na região do Rio Negro. Boletim do Museu Paraense Emílio Goeldi, Série Botânica, v. 8, p. 1-13, 1960.
VALVERDE, O. Estudo regional da Zona da Mata de Minas Gerais. Revista Brasileira de Geografia, v. 20, n. 1, p. $1-82,1958$.

VELOSO, H. P.; KLEIN, R. M. As comunidades e associações vegetais da mata pluvial do sul do Brasil. IV As associações situadas entre o rio Tubarão e a Lagoa dos Barros. Sellowia, v. 15, p. 57-114, 1963.

ZICKEL, C. S. Fitossociologia e dinâmica do estrato herbáceo de dois fragmentos florestais do estado de São Paulo. 1995. 129 f. Tese (Doutorado em Botânica) Campinas, Universidade Estadual de Campinas, Campinas, 1995. 